\title{
Subpoblaciones espermáticas en el semen de conejo refrigerado con diferentes diluyentes
}

\author{
Sperm subpopulations in chilled rabbit semen with different diluents \\ Camila Suárez A. ${ }^{1}$, Aarón Sierra P. ${ }^{1}$, Juan David Montoya P. ${ }^{2}$, \\ Giovanni Restrepo B. ${ }^{1,3}$
}

\section{Resumen}

La actividad reproductiva de diversas especies de mamíferos se encuentra influenciada por las subpoblaciones espermáticas de sus eyaculados. Dichas subpoblaciones se diferencian en las características de movilidad y cinética de los espermatozoides y han sido relacionadas y catalogadas según su capacidad para el proceso de fertilización. El objetivo de este estudio fue evaluar el efecto de la composición del diluyente de semen sobre las subpoblaciones espermáticas del semen refrigerado de conejo. Se obtuvo el semen de 10 conejos de la raza Californiano (tres eyaculados por animal). Cada eyaculado se dividió en cuatro alícuotas, las cuales se diluyeron en uno de cuatro diluyentes, cuya composición básica consistió en T1: leche descremada y azucares; T2: dextrosa, citrato sódico y acetato potásico; T3: caseinatos de sodio, fosfatos y azúcares; y T4: tris-ácido cítrico y yema de huevo. Cada alícuota se mantuvo en refrigeración a $16^{\circ} \mathrm{C}$ durante 72 horas. Inmediatamente después de la dilución y cada 24 horas se evaluó la distribución de cuatro subpoblaciones de espermatozoides mediante un sistema computarizado: Tipo A (espermatozoides rápidos progresivos), Tipo $\mathrm{B}$ (espermatozoides rápidos $\mathrm{y}$ espermatozoides medios progresivos), Tipo $\mathrm{C}$

\footnotetext{
${ }^{1}$ Facultad de Ciencias Agrarias, Universidad Nacional de Colombia, Medellín, Colombia

${ }^{2}$ Facultad de Ciencias Agrarias, Politécnico Colombiano Jaime Isaza Cadavid, Medellín, Colombia

${ }^{3}$ E-mail: grestre0@unal.edu.co
}

Recibido: 3 de marzo de 2021

Aceptado para publicación: 4 de septiembre de 2021

Publicado: 27 de octubre de 2021

CLos autores. Este artículo es publicado por la Rev Inv Vet Perú de la Facultad de Medicina Veterinaria, Universidad Nacional Mayor de San Marcos. Este es un artículo de acceso abierto, distribuido bajo los términos de la licencia Creative Commons Atribución 4.0 Internacional (CC BY 4.0) [https:// creativecommons.org/licenses/by/4.0/deed.es] que permite el uso, distribución y reproducción en cualquier medio, siempre que la obra original sea debidamente citada de su fuente original 
(espermatozoides medios y espermatozoides lentos), y Tipo D (espermatozoides estáticos). Los datos se analizaron mediante el PROC GLM y la prueba HSD de Tukey del software SAS v. 9.2. Se encontró que T1 y T3 produjeron resultados superiores para la velocidad curvilínea (VCL), la velocidad rectilínea (VSL), la velocidad promedio (VAP) y la amplitud de desplazamiento lateral de la cabeza (ALH) de los espermatozoides refrigerados durante 24 y 48 horas $(\mathrm{p}<0.05)$, mientras que $\mathrm{T} 2$ y T 4 produjeron un incremento en la frecuencia de batido (BCF) $(\mathrm{p}<0.05)$. Se concluye que la composición del diluyente influye sobre la distribución de las subpoblaciones espermáticas del semen refrigerado de conejo, al generar cambios en la progresividad y la cinética de los espermatozoides.

Palabras clave: espermatozoides, calidad seminal, cinética espermática, refrigeración

\section{AbSTRACT}

The reproductive activity of various species of mammals is influenced by the sperm subpopulations of their ejaculates. These subpopulations differ in the motility and kinetic characteristics of the sperm and have been related and classified according to their capacity for the fertilization process. The objective of this research was to evaluate the effect of the composition of the semen diluent on the sperm subpopulations of the chilled rabbit semen. The semen of 10 rabbits of the Californian breed (three ejaculated per animal) was obtained. Each ejaculate was divided into four aliquots, which were diluted in one of four diluents, whose basic composition consisted of T1: skim milk and sugars; T2: dextrose, sodium citrate and potassium acetate; T3: sodium caseinates, phosphates and sugars; and T4: tris-citric acid and egg yolk. Each aliquot was kept refrigerated at $16^{\circ} \mathrm{C}$ for $72 \mathrm{~h}$. Immediately after dilution and every $24 \mathrm{~h}$, the distribution of four sperm subpopulations was evaluated using a computerized system: Type A (rapid progressive spermatozoa), Type B (rapid spermatozoa and medium progressive spermatozoa), Type C (medium spermatozoa and slow spermatozoa) and Type D (static spermatozoa). The data were analyzed using the PROC GLM and Tukey's HSD test of the SAS v. 9.2. It was found that $\mathrm{T} 1$ and $\mathrm{T} 3$ produced superior results for curvilinear velocity (VCL), straight line velocity (VSL), average path velocity (VAP) and amplitude of lateral head displacement (ALH) of sperm refrigerated for 24 and 48 hours $(\mathrm{p}<0.05)$, while $\mathrm{T} 2$ and $\mathrm{T} 4$ produced an increase in the beat cross frequency (BCF) $(p<0.05)$. It is concluded that the composition of the diluent influences the distribution of the sperm subpopulations of the refrigerated rabbit semen, by generating changes in the progressivity and kinetics of the sperm.

Key words: sperm, semen quality, sperm kinetics, cooling

\section{INTRODUCCIÓN}

El estudio del semen de los mamíferos como una unidad, con una serie de características de calidad y movilidad definidas como un todo, ha sido reevaluado gracias a diversos estudios que demuestran la gran heterogeneidad que existe entre los esperma- tozoides, que se atribuyen a diferencias en los estados fisiológicos que transcurren durantelavida de estos (Núñez-Martínez et al., 2006; Maroto-Morales et al., 2012). Esto ha dado paso al estudio de las subpoblaciones espermáticas, que se encargan de clasificar un mismo eyaculado en varias secciones según sus propiedades, con el fin de determinar los espermatozoides más aptos para el pro- 
ceso de fertilización (Dorado et al., 2010; Bravo et al., 2011). Como resultado de esto, se ha evidenciado que la actividad reproductiva de diversas especies de mamíferos (Dorado et al., 2011, 2013; MarotoMorales et al., 2012; Vicente-Fiel et al., 2013; Estrada et al. 2017; Luna et al., 2017) se encuentra influenciada por las subpoblaciones espermáticas que presentan sus eyaculados.

El análisis, evaluación y clasificación de las subpoblaciones es un proceso que requiere de un sistema especial que permita obtener gran cantidad de información sobre las características de calidad, movilidad y morfología de una muestra de semen (BergsteinGalan et al., 2017). El Sistema de Análisis Asistido por Computadora (CASA) constituye uno de los métodos más confiables para realizar dicho estudio, según las investigaciones realizadas en las últimas décadas (Muiño et al., 2008; Luna et al., 2017).

Si bien, cada eyaculado presenta una serie de subpoblaciones con características definidas, existen procesos que pueden afectar su distribución. En particular, durante los procesos de refrigeración de semen, las principales variables que influyen en la distribución de las subpoblaciones son la temperatura, el tiempo y el diluyente utilizado (RodríguezGil et al., 2007; Dorado et al., 2010; Gallego et al., 2017). Estos pueden generar cambios en la calidad del eyaculado, afectando el resultado de la inseminación artificial (Souza et al., 2018). Así mismo, se han estudiado las implicaciones de la congelación y descongelación seminal sobre las subpoblaciones de espermatozoides de varias especies (Flores et al., 2008).

Existen pocos reportes sobre el estudio de las subpoblaciones espermáticas en conejos, mayormente relacionadas con la predicción de la fertilidad del semen y el tamaño de las camadas (Quintero-Moreno et al., 2007). Otro aspecto para considerar sería el posible efecto de la composición de los diluyentes utilizados. En conejos se reporta el uso de una variedad de diluyentes para la refrigeración del semen, entre estos, Tris, ácido cítrico, glucosa, fructosa, HEPES (ácido 4- [2hidroxietil] -1-piperazina-metanosulfónico) y yema de huevo (Roca et al., 2000; LópezGatius et al., 2005; Aksoy et al., 2010; ElSeadawy et al., 2017). Además, se tienen diluyentes comerciales basados en dextrosa, citrato sódico y acetato potásico (Suárez et al., 2020). Sin embargo, no se disponen de estudios sobre la influencia de la composición de los diluyentes en las subpoblaciones espermáticas de esta especie, así como, sobre la influencia de otros componentes que, como los caseinatos de sodio, han mostrado efectos favorables sobre varios parámetros cinéticos de los espermatozoides (Lagares et al., 2012; Martins et al., 2016). Por lo tanto, el objetivo de esta investigación fue evaluar el efecto de la composición del diluyente de semen sobre las subpoblaciones espermáticas del semen refrigerado de conejo.

\section{Materiales y Métodos}

\section{Animales y Muestras Seminales}

Se utilizaron 10 conejos (Oryctolagus cuniculus) machos de la raza Californiano, de 6 a 18 meses de edad. Los animales fueron alimentados dos veces al día, con una dieta a base de concentrado y forraje. Los animales tenían actividad reproductiva cada semana y fertilidad comprobada mediante el nacimiento de crías vivas. El semen se colectó con vagina artificial PLQ-135 (Pledge Agro, China), en presencia de una hembra para mejorar el estímulo sexual. Se obtuvieron tres eyaculados de cada macho. Se evaluó la movilidad individual de los espermatozoides mediante microscopía óptica. Solo se procesaron los eyaculados con una movilidad superior al 70\% (Mocé et al., 2010). El comité de ética del Politécnico Jaime Isaza Cadavid otorgó el aval en la categoría de riesgo mínimo según Resolución 8430 de 1993 (Aval CEPI 21052018). 


\section{Dilución y Refrigeración del Semen}

Cada eyaculado se dividió en cuatro alícuotas iguales, las cuales se diluyeron individualmente en uno de cuatro diluyentes (tratamientos), cuya composición básica consistió en T1: leche descremada y azucares (EquiPlus ${ }^{\circledR}$, Minitube, Alemania); T2: dextrosa, citrato sódico y acetato potásico (MRAbit $^{\mathbb{\circledR}}$, Kubus, España); T3: caseinatos de sodio, fosfatos y azúcares (PoliCryo, European Patent EP3169154B1); y T4: trisácido cítrico y yema de huevo (Triladyl ${ }^{\circledR}$, Minitube). La dilución se realizó a temperatura ambiente $\left(\sim 19^{\circ} \mathrm{C}\right)$ y el semen se llevó a una concentración final de $80 \times 10^{6}$ espermatozoides $/ \mathrm{ml}$. Una vez diluido, el semen se sometió a refrigeración a $16{ }^{\circ} \mathrm{C}$ durante 72 horas (Roca et al., 2000; LópezGatius et al., 2005).

\section{Evaluación de las Subpoblaciones Esper- máticas}

Las distribuciones de las subpoblaciones espermáticas fueron evaluadas después de la dilución y cada 24 horas utilizando el sistema Sperm Class Analyzer ( $\mathrm{SCA}^{\circledR}$ v. 5.1, Microptic, España) y un microscopio de contraste de fase (Eclipse E200, Nikon) con una cámara digital (Scout SCA780, Basler, USA). El software fue configurado para la especie con cubreobjetos de $20 \times 20 \mathrm{~mm}$, óptica en lente de ph-, gota de $7 \mu \mathrm{l}$ y platina térmica a $37^{\circ} \mathrm{C}$. Los parámetros evaluados fueron: velocidad rectilínea (VSL), velocidad curvilínea (VCL), velocidad media (VAP), amplitud lateral de la cabeza (ALH) y frecuencia de batido de la cola (BCF).

Se empleó la clasificación predeterminada por el software para la clasificación de las subpoblaciones espermáticas, según los criterios de la Organización Mundial de la Salud (OMS). Se establecieron cuatro tipos de espermatozoides: Tipo A (espermatozoides rápidos progresivos), Tipo $\mathrm{B}$ (espermatozoides rápidos y espermatozoides medios progresi- vos), Tipo C (espermatozoides medios y espermatozoides lentos), y Tipo D (espermatozoides estáticos). A partir de la VCL, los espermatozoides fueron clasificados como estáticos $(<15 \mu \mathrm{m} / \mathrm{s})$, lentos $(15-24 \mu \mathrm{m} / \mathrm{s})$, me$\operatorname{dios}(25-50 \mu \mathrm{m} / \mathrm{s})$ y rápidos $(>50 \mu \mathrm{m} / \mathrm{s})$. La progresividad fue definida como aquellos espermatozoides con un índice de rectitud $(\mathrm{STR})$ mayor a $70 \%$, donde STR $=(\mathrm{VSL} /$ VAP) x 100. En el análisis se incluyeron 1000 espermatozoides por cada eyaculado y tratamiento.

\section{Análisis Estadístico}

Se realizó mediante el ajuste de modelos lineales con la inclusión de los efectos fijos del conejo, el tiempo de refrigeración y de la interacción entre el tratamiento y la subpoblación espermática. Se calculó un índice de velocidad seminal (SVi) mediante el análisis de componentes principales (PCA) de los parámetros cinéticos de cada subpoblación espermática. Se extrajeron los eigenvectores para establecer la ecuación de SVi (Núñez-Martínez et al., 2006). Se evaluó la normalidad de los datos mediante la prueba de Shapiro-Wilk y las diferencias entre medias mediante la prueba de Tukey $(p<0.05)$. Todos los análisis se realizaron mediante el software SAS v. 9.2 (SAS, USA).

\section{Resultados}

El diluyente utilizado para la refrigeración seminal influyó sobre las proporciones de espermatozoides clasificados en diferentes subpoblaciones, según su velocidad y progresividad. Los efectos fijos del conejo, el tiempo de refrigeración y de la interacción entre el tratamiento y la subpoblación espermática fueron significativos para todos los modelos lineales ajustados para los diferentes parámetros seminales $(\mathrm{p}<0.0001)$. La Figura 1 muestra la distribución de las subpoblaciones espermáticas para cada diluyente y tiempo de refrigeración. 


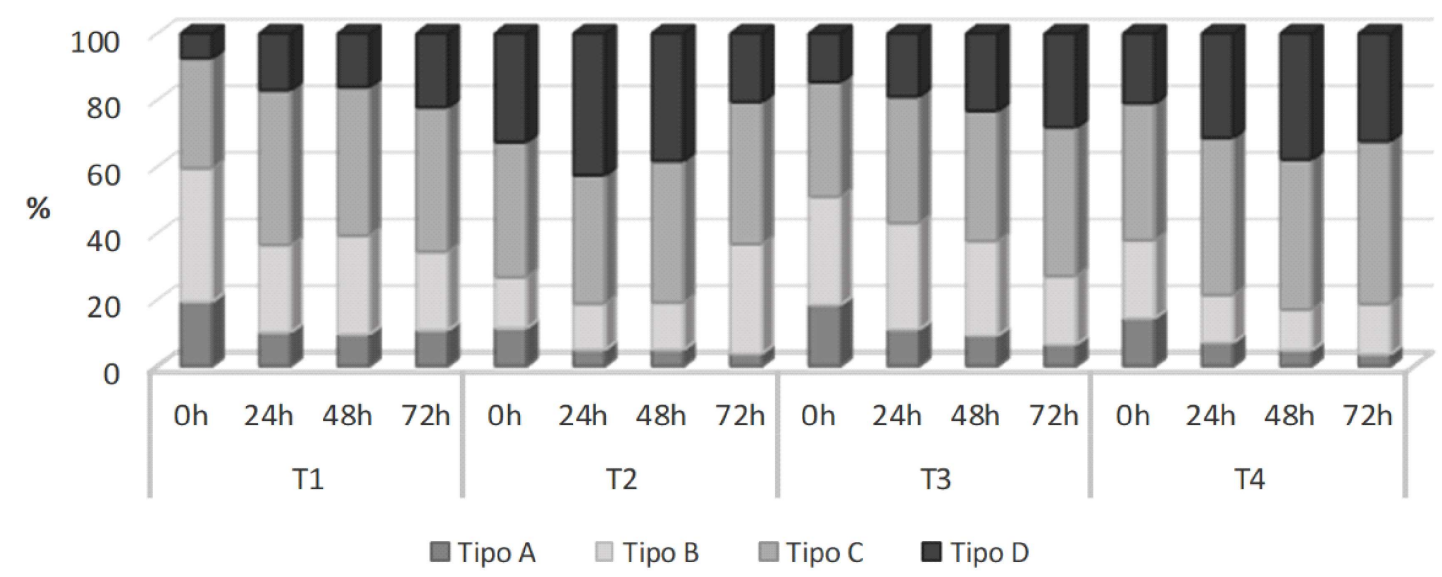

Figura 1. Distribución de subpoblaciones de espermatozoides en el semen de conejo según el diluyentes y tiempo de refrigeración T1: EquiPlus ${ }^{\circledR}$ (Minitube); T2: MRAbit ${ }^{\circledR}$ (Kubus); T3: PoliCryo; y T4: Triladyl ${ }^{(}$(Minitube).

Tipo A: espermatozoides rápidos progresivos. Tipo $\mathrm{B}$ : espermatozoides rápidos $\mathrm{y}$ espermatozoides medios progresivos. Tipo C: espermatozoides medios y espermatozoides lentos. Tipo D: espermatozoides estáticos

Cuadro 1. Parámetros cinéticos del semen de conejo por tratamiento después de la dilución (hora 0 )

\begin{tabular}{ccccc}
\hline Parámetro & T1 & T2 & T3 & T4 \\
\hline VCL & $59.35 \pm 3.64^{\mathrm{a}}$ & $44.41 \pm 5.71^{\mathrm{b}}$ & $56.05 \pm 4.32^{\mathrm{a}}$ & $44.15 \pm 3.94^{\mathrm{b}}$ \\
VAP & $38.86 \pm 2.67^{\mathrm{a}}$ & $29.71 \pm 4.60^{\mathrm{b}}$ & $37.32 \pm 3.75^{\mathrm{ab}}$ & $28.96 \pm 3.61^{\mathrm{b}}$ \\
VSL & $25.30 \pm 2.38^{\mathrm{a}}$ & $21.48 \pm 2.79^{\mathrm{a}}$ & $24.11 \pm 2.85^{\mathrm{a}}$ & $20.75 \pm 2.97^{\mathrm{a}}$ \\
ALH & $2.73 \pm 0.09^{\mathrm{a}}$ & $2.23 \pm 0.15^{\mathrm{b}}$ & $2.78 \pm 0.09^{\mathrm{a}}$ & $2.43 \pm 0.12^{\mathrm{ab}}$ \\
BCF & $6.58 \pm 0.22^{\mathrm{a}}$ & $7.31 \pm 0.66^{\mathrm{a}}$ & $6.72 \pm 0.27^{\mathrm{a}}$ & $7.26 \pm 0.64^{\mathrm{a}}$ \\
SVi & $0.62 \pm 0.003$ & $0.45 \pm 0.005$ & $0.60 \pm 0.003$ & $0.51 \pm 0.004$ \\
\hline
\end{tabular}

T1: EquiPlus ${ }^{\circledR}$ (Minitube); T2: MRAbit ${ }^{\circledR}$ (Kubus); T3: PoliCryo; y T4: Trilady ${ }^{\circledR}$ (Minitube) VCL: Velocidad curvilínea. VSL: Velocidad rectilínea. VAP: Velocidad promedio. ALH: Amplitud lateral de la cabeza. BCF: Frecuencia batido de cola. SVi: índice de velocidad seminal Los resultados están expresados como media \pm error estándar. Superíndices diferentes dentro de filas indican diferencias significativas entre tratamientos $(p<0.05)$

La dilución del semen en cada tratamiento generó una respuesta variable en los parámetros cinéticos de los espermatozoides. Se calculó el SVi como una forma de expresar integralmente la cinética espermática (Cuadro 1).
Las subpoblaciones espermáticas establecidas presentaron variaciones en la magnitud de los parámetros cinéticos evaluados. En la Figuras 2, 3 y 4 se presentan los resultados de las velocidades espermáticas (VCL, VSL y VAP) para las subpoblaciones 
de espermatozoides de los tipos A, B y C, respectivamente.

La ALH mostró de manera reiterada resultados mayores para $\mathrm{T} 1$ y $\mathrm{T} 3$ en los diferentes tiempos de refrigeración y subpoblaciones (Figura 5). La BCF presentó valores mayores para T2 y T4 en la subpoblación de espermatozoides tipo A a las 0,24 y $48 \mathrm{~h}$ de refrigeración (Figura 6), mientras que no se observó una tendencia concreta en las diferencias entre los tratamientos para las subpoblaciones de tipo B y C.

\section{Discusión}

Diferentes investigaciones han reportado subpoblaciones en el semen de mamíferos que muestran la heterogeneidad de los espermatozoides presentes dentro de una muestra (Quintero-Moreno et al., 2007; Santolaria et al., 2015). Esta característica no necesariamente se atribuye a factores externos (Mendoza et al., 2012); por lo tanto, la explicación para la presencia de diferentes tipos de espermatozoides dentro de una misma muestra de semen se ha asociado con los estadios de vida o maduración de los espermatozoides (Dorado et al., 2013). Así mismo, las subpoblaciones podrían representar diferentes momentos fisiológicos en las células espermáticas, lo que podría constituirse como una estrategia reproductiva relacionada con la capacitación, que facilite alcanzar el éxito reproductivo mediante la fertilización (Valverde Abarca y Madrigal Valverde, 2018). En el presente estudio se evidenciaron diferentes subpoblaciones espermáticas, catalogadas de acuerdo con parámetros específicos de su movilidad y cinética, y con diferente distribución según el tiempo de almacenamiento en refrigeración. A su vez, el diluyente mostró un efecto determinante en la distribución de dichas subpoblaciones, siendo evidente la reducción de las poblaciones de espermatozoides estáticos (Tipo D) con el uso de T1 y T3 (Figura 1). Quintero-Moreno et al. (2007) clasificaron las subpoblaciones de espermatozoides de conejo según su movilidad, encontrando dos subpoblaciones con movimiento progresivo y dos con movimiento no lineal; siendo las primeras, aquellas relacionadas con menor proporción de anormalidades y menos cromosomas alterados en los espermatozoides.

Los parámetros cinéticos evaluados al momento de la dilución, así como el SVi estimado (Cuadro 1), muestran que la composición del diluyente influye sobre las características de movimiento de los espermatozoides, posiblemente por un efecto directo sobre la fisiología y el metabolismo espermático. Así mismo, se evidenció que las velocidades de desplazamiento fueron superiores para la subpoblación de espermatozoides Tipo A (Figura 2), en comparación con las subpoblaciones Tipo B (Figura 3) y Tipo C (Figura 4). Se ha reportado diversas formas de modificar las subpoblaciones en su distribución y cantidad; por ejemplo, con la adición de algún compuesto (Ramió et al., 2008; Kanuga et al., 2012), la composición de proteínas del plasma seminal (Ledesma et al., 2017), con procesos como tinción o centrifugación (Miró et al., 2009; D’Amours et al. 2012) o mediante congelación o refrigeración durante tiempos específicos (Dorado et al., 2011). En este sentido, tanto la composición del diluyente como el tiempo en refrigeración produjeron diferencias en el comportamiento de las subpoblaciones espermáticas, tanto en la velocidad de desplazamiento como en parámetros asociados con la capacitación, la hiperactivación y la capacidad fertilizante de los espermatozoides como ALH (Figura 5) y BCF (Figura 6).

En este estudio se encontró que la refrigeración del semen de conejo a $16^{\circ} \mathrm{C}$ permite conservar las características de movimiento de los espermatozoides por 72 horas, de forma más eficiente con los diluyentes compuestos por leche descremada y azucares o por caseinatos de sodio, fosfatos y azúcares, en comparación con aquellos basados en dextrosa, citrato sódico y acetato potásico o en tris- 


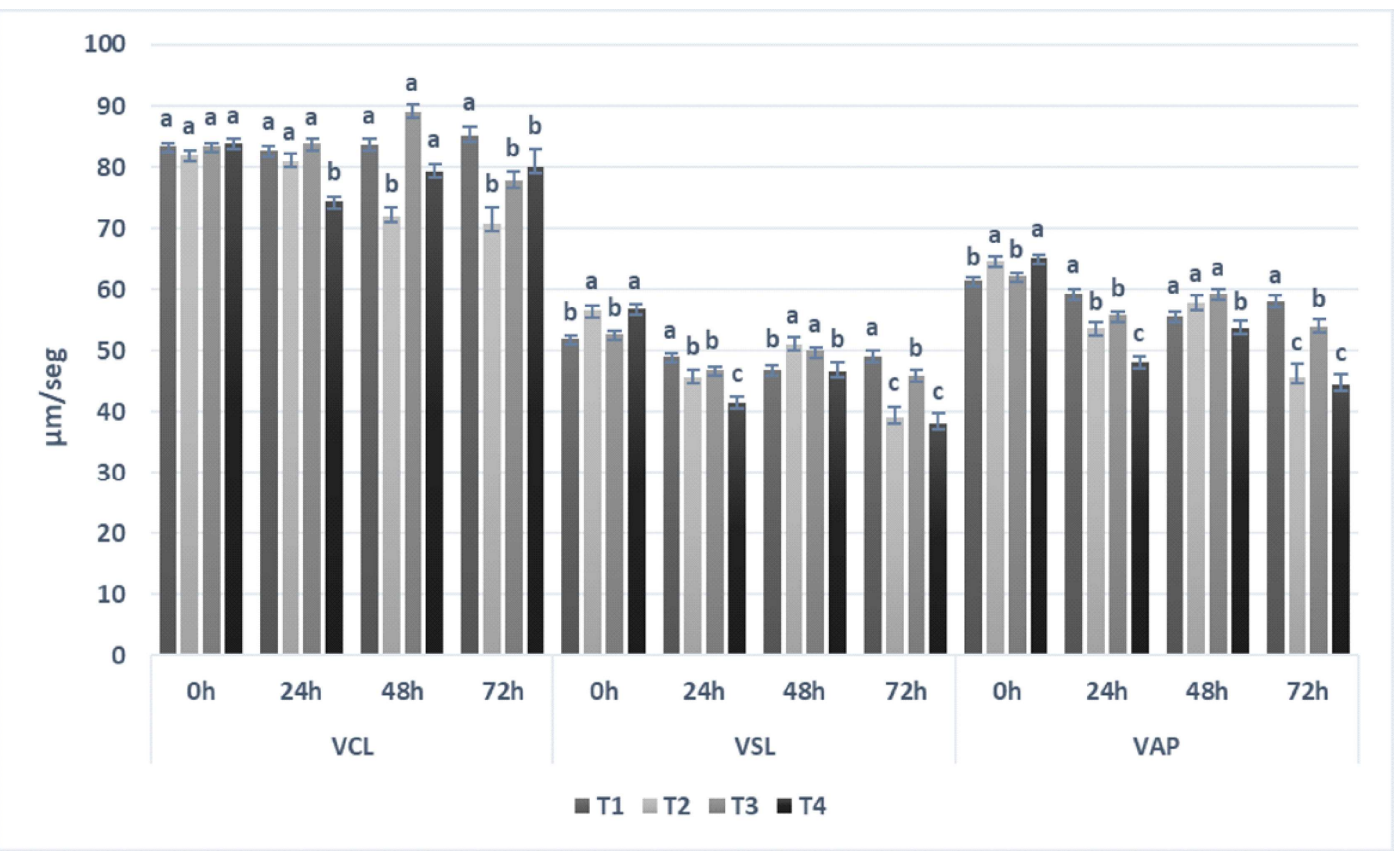

Figura 2. Velocidades espermáticas de la subpoblación Tipo A por diluyente y tiempo de refrigeración.

T1: EquiPlus ${ }^{\circledR}$ (Minitube); T2: MRAbit ${ }^{\circledR}$ (Kubus); T3: PoliCryo; y T4: Triladyl ${ }^{\circledR}$ (Minitube)

VCL: Velocidad curvilínea. VSL: Velocidad rectilínea. VAP: Velocidad promedio

Los resultados están expresados como media \pm error estándar. Letras diferentes indican diferencia significativa entre tratamientos $(\mathrm{p}<0.05)$

ácido cítrico y yema de huevo. No obstante, está última composición presentó al momento de la dilución el tercer mayor valor para el $\mathrm{SVi}$, aunque en la mayoría de los casos produjo la reducción más severa de los parámetros cinéticos en las diferentes subpoblaciones espermáticas. El-Seadawy et al. (2017) encontraron que la suplementación del diluyente trisácido cítrico-glicerol y yema de huevo con extracto etanólico de propóleo (PEE) fue capaz de mantener las características del semen refrigerado de conejo durante las $72 \mathrm{~h}$.
De acuerdo con los hallazgos de este estudio, la clasificación e identificación de las subpoblaciones en el semen de conejo podría tener implicaciones prácticas en los programas de inseminación artificial; así mismo, según otros autores, la estructura de las subpoblaciones de espermatozoides móviles podría desempeñar un papel importante en la determinación de la capacidad fertilizante en muestras con un índice bajo de fertilización in vivo (Quintero-Moreno et al., 2007). 


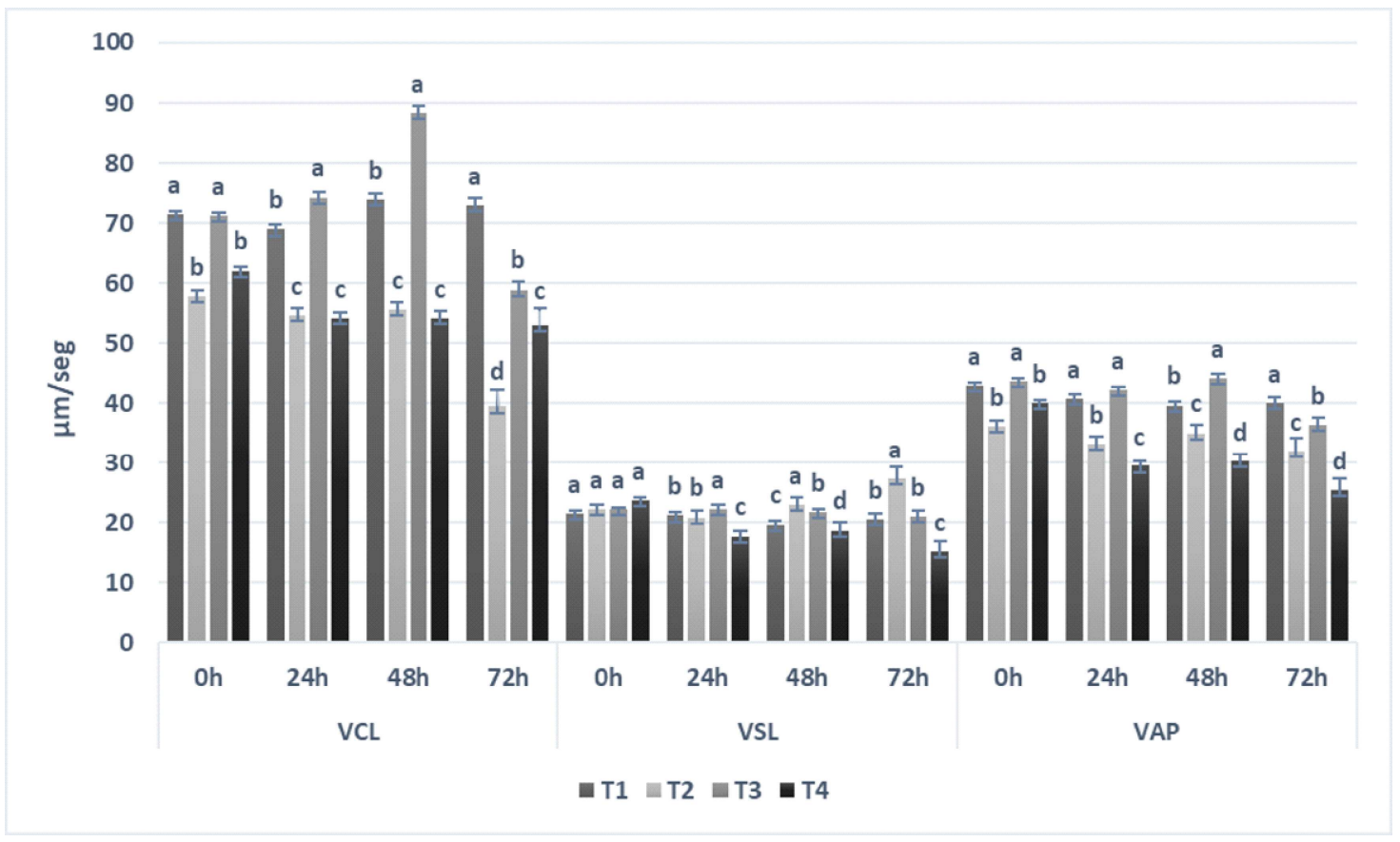

Figura 3. Velocidades espermáticas de la subpoblación Tipo B por diluyente y tiempo de refrigeración.

T1: EquiPlus ${ }^{\circledR}$ (Minitube); T2: MRAbit ${ }^{\circledR}$ (Kubus); T3: PoliCryo; y T4: Triladyl ${ }^{\circledR}$ (Minitube)

VCL: Velocidad curvilínea. VSL: Velocidad rectilínea. VAP: Velocidad promedio Los resultados están expresados como media \pm error estándar. Letras diferentes indican diferencia significativa entre tratamientos $(\mathrm{p}<0.05)$

\section{Conclusiones}

- Los diluyentes compuestos por leche descremada y azúcares o por caseinatos de sodio, fosfatos y azúcares son una alternativa apropiada para la conservación de semen de conejo en condiciones de refrigeración, dado que favorecen el mantenimiento prolongado de los parámetros cinéticos de los espermatozoides y la prevalencia de subpoblaciones espermáticas con características superiores de velocidad y progresividad.
- La composición del diluyente para la refrigeración afecta en la distribución y las características cinéticas del semen de conejo y, por lo tanto, podría influir en su capacidad fertilizante.

\section{Agradecimientos}

A la Universidad Nacional de Colombia por su apoyo financiero y al personal del laboratorio de andrología y de la granja Román Gómez Gómez del Politécnico Colombiano Jaime Isaza Cadavid. 


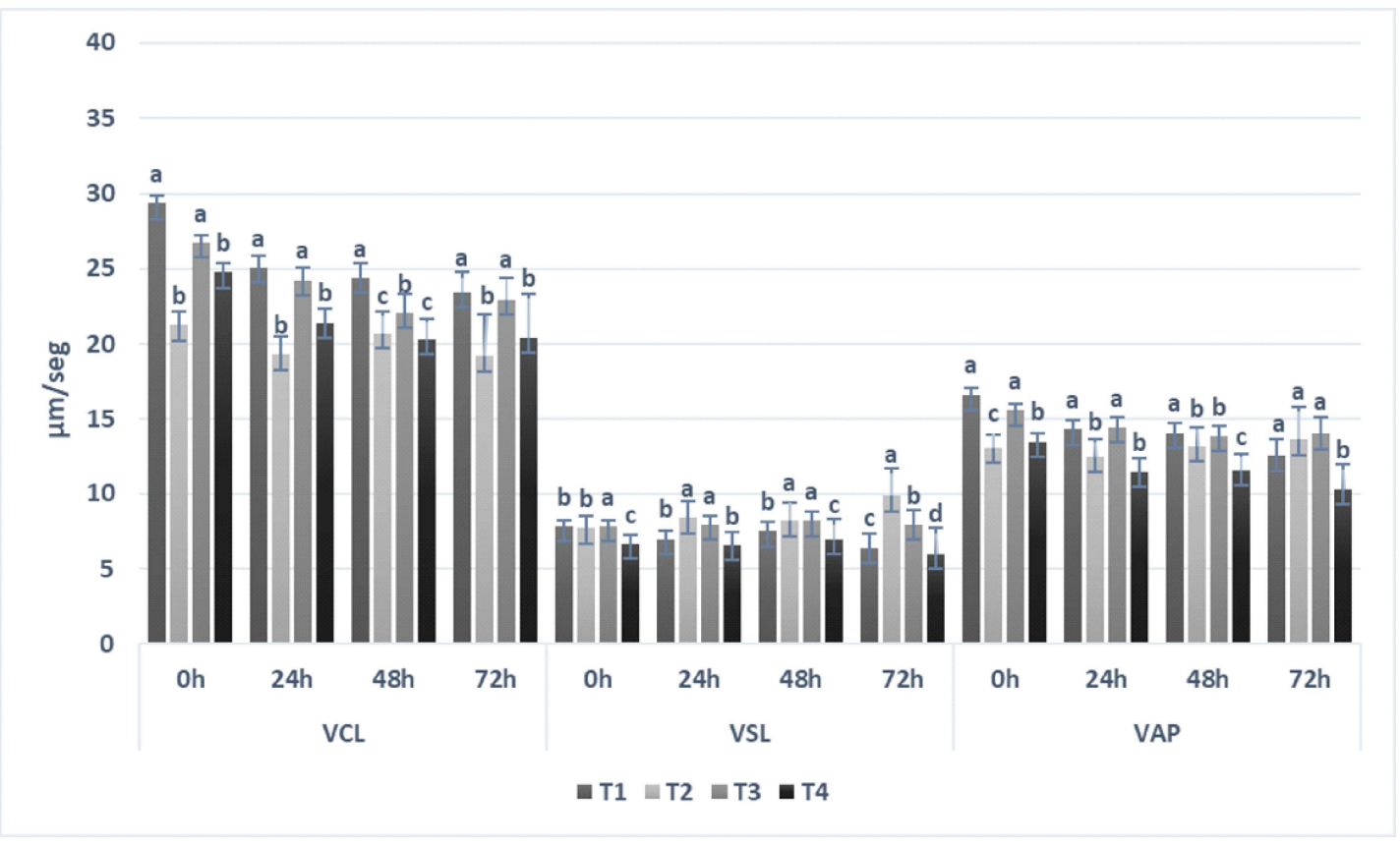

Figura 4. Velocidades espermáticas de la subpoblación Tipo C por diluyente y tiempo de refrigeración.

T1: EquiPlus ${ }^{\circledR}$ (Minitube); T2: MRAbit ${ }^{\circledR}$ (Kubus); T3: PoliCryo; y T4: Triladyl ${ }^{\circledR}$ (Minitube)

VCL: Velocidad curvilínea. VSL: Velocidad rectilínea. VAP: Velocidad promedio

\section{Literatura Citada}

1. Aksoy M, Cankat Lehimcioglu N, Akman O. 2010. Effect of seminal plasma on functional integrity of rabbit sperm membranes during storage at $4{ }^{\circ} \mathrm{C}$ or freezing. World Rabbit Sci 16: 1115-1122. doi: 10.4995/wrs.2008.642

2. Bergstein-Galan TG, Weiss RR, Kozicki LE, Bicudo SD. 2017. Sperm subpopulations in ejaculated sperm and spermatozoa recovered from ovine epididymides up to $48 \mathrm{~h}$ after death. Anim Reprod Sci 187: 20-27. doi: 10.1016/ j.anireprosci.2017.10.001

3. Bravo JA, Montanero J, Calero $R$, Roy TJ. 2011. Identification of sperm subpopulations with defined motility characteristics in ejaculates from Ile de France rams. Anim Reprod Sci 129: 2229. doi:10.1016/j.anireprosci.2011.10.005
4. D'Amours O, Bordeleau LJ, Frenette G, Blondin P, Leclerc P, Sullivan R. 2012. Binder of sperm 1 and epididymal sperm binding protein 1 are associated with different bull sperm subpopulations. Reproduction 143:759-771. doi: 10.1530/ REP-11-0392

5. Dorado J, Acha D, Ortiz I, Gálvez MJ, Carrasco JJ, Díaz B, Gómez-Arrones V, Calero-Carretero R, Hidalgo M. 2013. Relationship between conventional semen characteristics, sperm motility patterns and fertility of Andalusian donkeys (Equus asinus). Anim Reprod Sci 143: 64-71. doi: 10.1016/j.anireprosci.2013.10.003

6. Dorado J, Alcaráz L, Duarte N, Portero JM, Acha D, Hidalgo M. 2011. Changes in the structures of motile sperm subpopulations in dog spermatozoa after both cryopreservation and centrifugation 


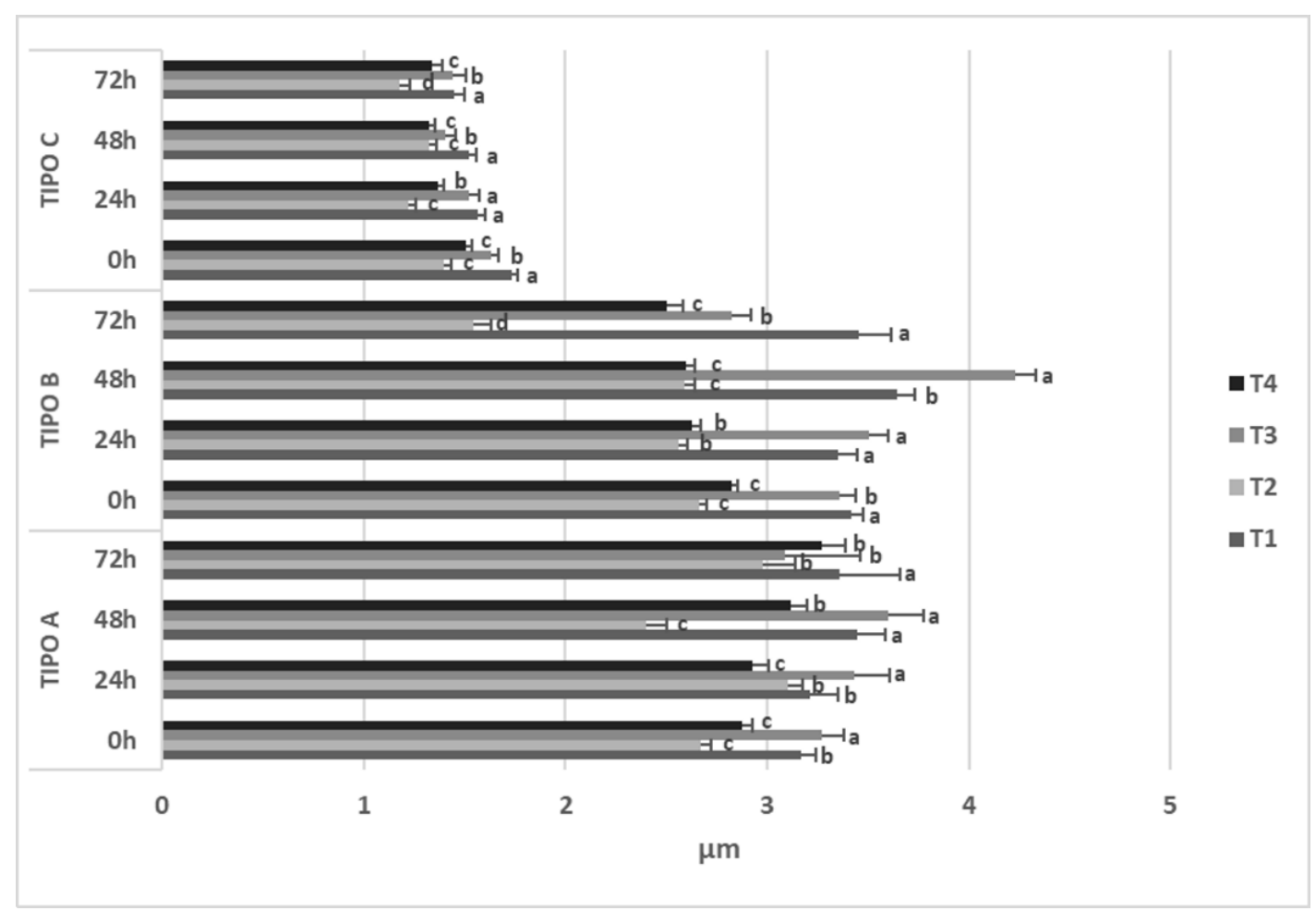

Figura 5. Amplitud lateral de la cabeza (ALH) de las subpoblaciones espermáticas por diluyente y tiempo de refrigeración.

T1: EquiPlus ${ }^{\circledR}$ (Minitube); T2: MRAbit ${ }^{\circledR}$ (Kubus); T3: PoliCryo; y T4: Triladyl ${ }^{\circledR}$ (Minitube)

VCL: Velocidad curvilínea. VSL: Velocidad rectilínea. VAP: Velocidad promedio Tipo A: espermatozoides rápidos progresivos. Tipo B: espermatozoides rápidos y espermatozoides medios progresivos. Tipo C: espermatozoides medios y espermatozoides lentos.

Los resultados están expresados como media \pm error estándar. Letras diferentes indican diferencia significativa entre tratamientos $(\mathrm{p}<0.05)$.

on PureSperm ${ }^{\circledR}$ gradient. Anim Reprod Sci 125: 211-218. doi: 10.1016/j.anireprosci.2011.03.013

7. Dorado J, Muñoz-Serrano A, Hidalgo M. 2010. The effect of cryopreservation on goat semen characteristics related to sperm freezability. Anim Reprod Sci 121: 115-123. doi: 10.1016/ j.anireprosci.2010.04.182

8. El-Seadawy IES, El-Nattat WS, ElTohamy MM, Aziza SAH, El-Senosy YA, Hussein AS. 2017. Preservability of rabbit semen after chilled storage in tris based extender enriched with different concentrations of Propolis ethanolic extract (PEE). Asian Pac J Reprod 6: 68-76. doi: 10.12980/apjr.6.20170204

9. Estrada E, Rivera del Álamo MM, Rodríguez-Gil JE, Yeste M. 2017. The addition of reduced glutathione to cryopreservation media induces changes in the structure of motile subpopulations of frozen-thawed boar sperm. Cryobio- 


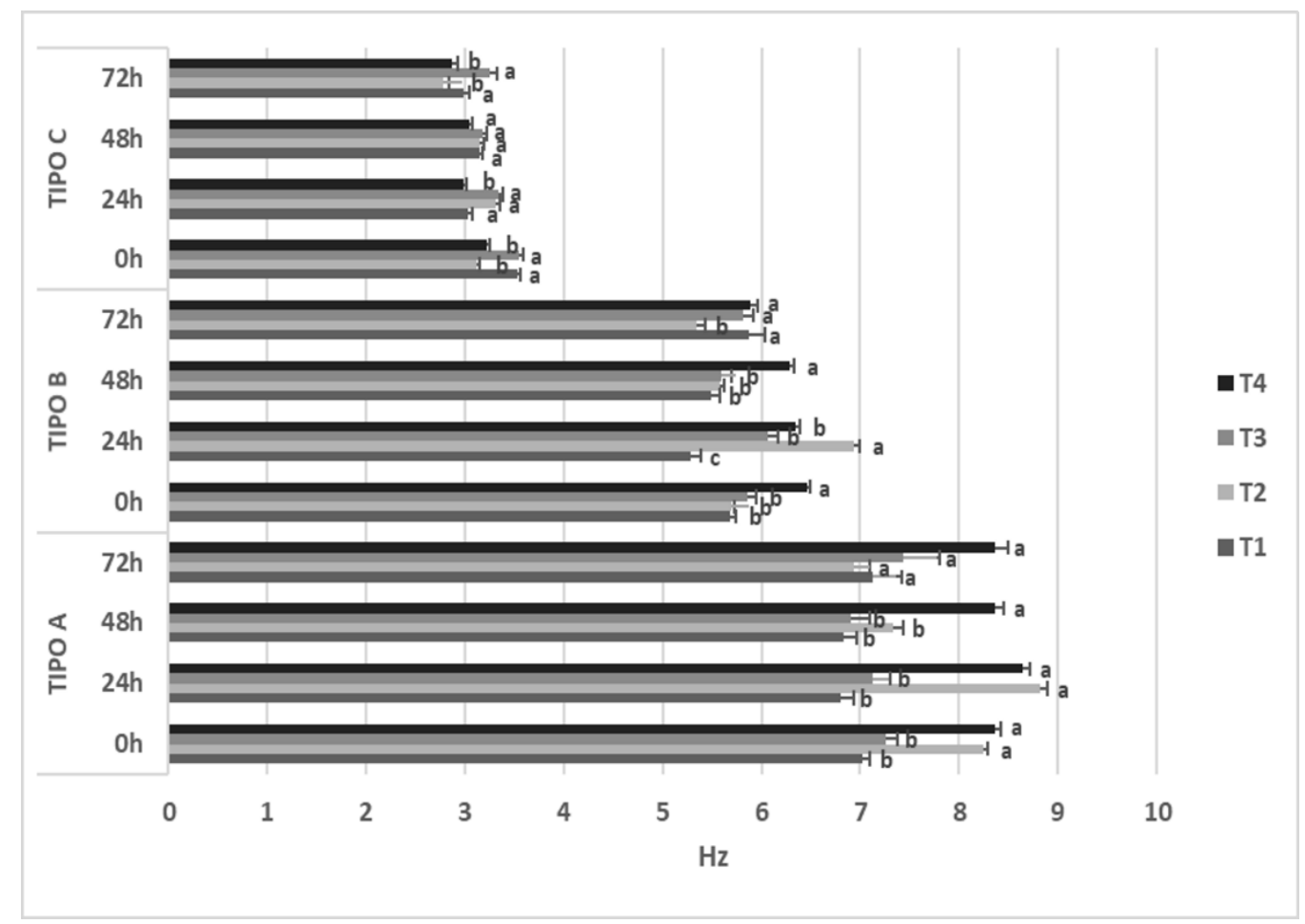

Figura 6. Frecuencia de batido de cola $(\mathrm{BCF})$ de las subpoblaciones espermáticas por diluyente y tiempo de refrigeración.

T1: leche descremada y azucares (EquiPlus ${ }^{\circledR}$, Minitube, Alemania). T2: dextrosa, citrato sódico y acetato potásico (MRAbit ${ }^{\circledR}$, Kubus, España). T3: caseinatos de sodio, fosfatos y azúcares (PoliCryo, European Patent EP3169154B1). T4: trisácido cítrico y yema de huevo (Triladyl ${ }^{\circledR}$, Minitube, Alemania)

Tipo A: espermatozoides rápidos progresivos. Tipo $\mathrm{B}$ : espermatozoides rápidos $\mathrm{y}$ espermatozoides medios progresivos. Tipo C: espermatozoides medios y espermatozoides lentos

Los resultados están expresados como media \pm error estándar. Letras diferentes indican diferencia significativa entre tratamientos $(\mathrm{p}<0.05)$.

logy 78: 56-64. doi: 10.1016/j.cryobiol.2017.07.002

10. Flores E, Taberner E, Rivera MM, Peña A, Rigau T, Miró J, RodríguezGil JE. 2008. Effects of freezing/ thawing on motile sperm subpopulations of boar and donkey ejaculates. Theriogenology 70: 936-945. doi: 10.1016/ j.theriogenology.2008.05.056
11. Gallego V, Cavalcante SS, Fujimoto RY, Carneiro PCF, Azevedo HC, Maria $A N$. 2017. Fish sperm subpopulations: changes after cryopreservation process and relationship with fertilization success in tambaqui (Colossoma macropomum). Theriogenology 87: 1624. doi: 10.1016/j.theriogenology.2016.08.001 
12. Kanuga MK, Drew RE, Wilson-Leedy JG, Ingermann RL. 2012. Subpopulation distribution of motile sperm relative to activation medium in steelhead (Oncorhynchus mykiss). Theriogenology 77: 916-925. doi: 10.1016/j.theriogenology.2011.09.020

13. Lagares MA, Martins HS, Carvalho IA, Oliveira CA, Souza MR, Penna CFAM, Cruz BC, et al. 2012. Caseinate protects stallion sperm during semen cooling and freezing. Cryo-Lett 33 : 214-219.

14. Ledesma A, Zalazar L, FernándezAlegre E, Hozbor F, Cesari A, Martínez-Pastor F. 2017. Seminal plasma proteins modify the distribution of sperm subpopulations in cryopreserved semen of rams with lesser fertility. Anim Reprod Sci 184: 44-50. doi: 10.1016/ j.anireprosci.2017.06.015

15. López-Gatius F, Sances G, Sancho M, Yániz J, Santolaria P, Gutiérrez R, Núñez M, et al. 2005. Effect of solid storage at $15^{\circ} \mathrm{C}$ on the subsequent motility and fertility of rabbit semen. Theriogenology 64: 252-260. doi: 10.1016/j.theriogenology.2004.11.015

16. Luna C, Yeste M, Rivera Del Alamo MM, Domingo J, Casao A, RodriguezGil JE, Pérez-Pé R, et al. 2017. Effect of seminal plasma proteins on the motile sperm subpopulations in ram ejaculates. Reprod Fert Develop 29: 394-405. doi: 10.1071/RD15231

17. Maroto-Morales A, Ramón M, GarcíaÁlvarez O, Soler AJ, Fernández-Santos MR, Roldan ERS, Gomendio M, et al. 2012. Morphometrically-distinct sperm subpopulations defined by a multistep statistical procedure in Ram ejaculates: Intra- and interindividual variation. Theriogenology 77: 1529-1539. doi: 10.1016/j.theriogenology.2011.11.020

18. Martins HS, Souza MR, Penna CFAM, da Silva GC, Côrtes SF, Stahlberg R, Lagares MA. 2016. Milk, caseinate and lactoferrin addition to equine semen cooling extenders. Andrologia 48: 862-868. doi: 10.1111/and.12523
19. Mendoza N, Casao A, Del Valle I, Serrano E, Nicolau S, Asumpção MEOA, Muiño-Blanco T, et al. 2012. Quality characteristics and fertilizing ability of ram sperm subpopulations separated by partition in an aqueous two-phase system. J Chromatogr B 880: 74-81. doi: 10.1016/j.jchromb.2011.11.019

20. Miró J, Taberner E, Rivera M, Peña $A$, Medrano A, Rigau T, Peñalba A. 2009. Effects of dilution and centrifugation on the survival of spermatozoa and the structure of motile sperm cell subpopulations in refrigerated Catalonian donkey semen. Theriogeno-logy 72 : 1017-1022. doi: 10.1016/j.theriogenology.2009.06.012

21. Mocé E, Lavara R, Vicente JS. 2010. Effect of cooling rate to $5{ }^{\circ} \mathrm{C}$, straw size and farm on fertilizing ability of cryopreserved rabbit sperm. Reprod Domest Anim 45: e1-7 doi: 10.1111/ j.1439-0531.2009.01507.x

22. Muiño R, Tamargo C, Hidalgo CO, Peña AI. 2008. Identification of sperm subpopulations with defined motility characteristics in ejaculates from Holstein bulls: dffects of cryopreservation and between bull variation. Anim Reprod Sci 109: 27-39. doi: 10.1016/j.anireprosci.2007.10.007

23. Núñez-Martínez I, Moran JM, Peña FJ. 2006. A three-step statistical procedure to identify sperm kinematic subpopulations in canine ejaculates: changes after cryopreservation. Reprod Domest Anim 41: 408-415. doi: 10.1111/ j.1439-0531.2006.00685.x

24. Quintero-Moreno A, Rigau T, Rodríguez-Gil JE. 2007. Multivariate cluster analysis regression procedures as tools to identify motile sperm subpopulations in rabbit semen and to predict semen fertility and litter size. Reprod Domest Anim 42: 312-319. doi: 10.1111/ j.1439-0531.2006.00785.x

25. Ramió L, Rivera MM, Ramírez A, Concha II, Peña A, Rigau T, Rodríguez-Gil JE. 2008. Dynamics of motile-sperm subpopulation structure in 
boar ejaculates subjected to «in vitro» capacitation and further «in vitro» acrosome reaction. Theriogenology 69: 501-512. doi: 10.1016/j.theriogenology.2007.10.021

26. Roca J, Martínez S, Vázquez JM, Lucas X, Parrilla I, Martínez EA. 2000. Viability and fertility of rabbit spermatozoa diluted in Tris-buffer extenders and stored at $15{ }^{\circ} \mathrm{C}$. Anim Reprod Sci 64: 103-112. doi: 10.1016/ S0378-4320(00)00185-8

27. Rodríguez-Gil JE, Silvers G, Flores E, Jesús Palomo $M$, Ramírez $A$, Montserrat Rivera M, et al. 2007. Expression of the GM-CSF receptor in ovine spermatozoa: GM-CSF effect on sperm viability and motility of sperm subpopulations after the freezing-thawing process. Theriogenology 67: 1359-1370. doi: 10.1016/j.theriogenology.2007.02.008

28. Santolaria P, Vicente-Fiel S, Palacín I, Fantova E, Blasco ME, Silvestre MA, Yániz JL. 2015. Predictive capacity of sperm quality parameters and sperm subpopulations on field fertility after artificial insemination in sheep.
Anim Reprod Sci 163: 82-88. doi:10.1016/j.anireprosci.2015.10.001

29. Souza AK, Trautwein LGC, Paranzini CS, Perencin FM, Cardoso GS, Martins MIM. 2018. Influence of cooling temperature in sperm subpopulations of domestic cats. Anim Reprod Sci 189: 1-10. doi: 10.1016/j.anireprosci.2017.12.015

30. Suárez C, Sierra A, Restrepo D, Duque JE, Restrepo G. 2020. Evaluación de diluyentes para la refrigeración de semen de conejo (Oryctolagus cuniculus). Rev Inv Vet Perú 31: 17857. doi: 10.15381/rivep.v31i2.17857

31. Valverde Abarca A, Madrigal Valverde M. 2018. Sistemas de análisis computa-dorizado de semen en la reproducción animal. Agron Mesoam 29: 469. doi: 10.15517/ma.v29i2.30613

32. Vicente-Fiel S, Palacín I, Santolaria P, Yániz JL. 2013. A comparative study of sperm morphometric subpopulations in cattle, goat, sheep and pigs using a computer-assisted fluorescence method (CASMA-F). Anim Reprod Sci 139: 182-189. doi: 10.1016/j.anireprosci.2013.04.002 